\section{First day post-operative review following uncomplicated phacoemulsification}

\begin{abstract}
Purpose To determine whether the first day review is essential in management of patients following uncomplicated phacoemulsification with intraocular lens implantation. Patients are routinely seen on the day following surgery. This can produce logistical problems in patient attendance that can necessitate an overnight stay. If the first day review were abandoned this would lead to an increased uptake of day case surgery and a reduction in health care costs.

Methods A retrospective cohort study was performed on all cases of uncomplicated phacoemulsification with intraocular lens implant surgery over a 6 month period. Slit lamp examination findings on the first postoperative day were reviewed. Visual acuity, corneal clarity, anterior chamber activity, intraocular pressure and configuration of the pupil were recorded. Any cases that failed to meet predetermined criteria underwent full case-note review. The subsequent
\end{abstract} management of these patients was analysed. Results Of the 201 cases of uncomplicated surgery, 74 cases $(37 \%)$ failed to meet the study criteria and underwent review. Of these only $12(6 \%)$ had their management altered as a result of the first day post-operative findings. This was entirely due to raised intraocular pressure. Visual acuity, corneal oedema and activity in the anterior chamber all improved on subsequent follow-up.

Conclusions The first day review of uncomplicated phacoemulsification with lens implant surgery provides the opportunity to treat raised intraocular pressure. A prospective randomised study is needed to identify means to prevent the post-operative intraocular pressure rise before we are able to consider abandoning the first review.

Key words Audit, Cataract surgery, Day case surgery, Follow-up, Intraocular pressure, Phacoemulsification, Post-operative review

Victoria M.L. Cohen

Department of

Ophthalmology

Royal Hallamshire Hospital

8 Beech Hill Road

Sheffield S10 2SB, UK

The advantages of small-incision surgery and intraoperative maintenance of intraocular pressure have led to a reduction in the number
V.M.L. COHEN, H. DEMETRIA,

K. JORDAN, R.J. LAMB, A.J. VIVIAN and type of post-operative complications following cataract surgery. ${ }^{1}$ With continued demand for time management and cost efficiency, the popularity of day case surgery has rapidly increased. Phacoemulsification has been shown to be suitable as a day case procedure. $^{2}$

There has been much debate about the frequency and length of follow-up after phacoemulsification. The Royal College of Ophthalmologists guidelines issued in 1995 suggest review within $48 \mathrm{~h}$ of surgery ${ }^{3}$ and hence most centres have adopted review on the first day following cataract extraction. This often poses difficulties for elderly patients. The first day review contributes to a reduced uptake of day case surgery and higher costs to the hospital service. ${ }^{4}$ Therefore, a retrospective study was performed to ascertain whether findings at the first post-operative review led to a change in management of the patient.

\section{Methods}

All cases of phacoemulsification and lens implant performed between 1 January and 30 June 1996 were identified. Operations performed by consultants and juniors (below consultant grade) were included. Both day case and overnight stay surgery were included. Cases were performed under local anaesthetic, which was administered by sub-Tenon's, peribulbar or retrobulbar technique. A sutureless corneal incision of 3-5 mm was employed. The continuous circular capsulorhexis was performed under viscoelastic (sodium hyaluronate 1\%).

Phacoemulsification was performed followed by aspiration of the soft lens matter and in-the-bag placement of a silicone optic, foldable intraocular lens of appropriate refractive power. Finally careful removal of the viscoelastic substance at the end of surgery and an orbital floor or subconjunctival injection of betamethasone and cefuroxime was administered.

Standard post-operative management involved removal of the eye shield the following morning, slit lamp examination and the prescription of combined betnesol and 
neomycin eye drops three times a day. Refraction was performed at the final clinic appointment 3 weeks after surgery.

\section{Exclusion criteria}

The following criteria were used to define complicated surgery:

Pre-operative: Previous intraocular surgery, planned phacotrabeculectomy, current glaucoma treatment and previous uveitis.

Per-operative: Posterior capsule tear, vitreous loss, zonule dehiscence, remaining soft lens matter and conversion to extracapsular cataract extraction.

All cases with complicated surgery were excluded from the study.

\section{Examination criteria}

The examination findings on the first day following surgery were recorded. Five predetermined examination criteria were chosen, as listed below. Any patient whose post-operative findings failed one or more of the examination criteria was subject to case-note review. The management of these cases was studied to assess whether the first day review altered the subsequent treatment of the patient.

1. Improvement or no change in best corrected visual acuity at day 1 .

2. A clear central cornea.

3. Less than $2+$ of cells in the anterior chamber.

4. No eccentricity of the pupil.

5. Intraocular pressure of less than $30 \mathrm{mmHg}$.

\section{Results}

Five hundred cataracts were extracted by phacoemulsification over this 6 month period. Of these, 201 cases were determined to be uncomplicated according to pre- and per-operative exclusion criteria as defined in Methods. Thirty-three patients who did not have slit lamp examination on the first post-operative day were excluded from the study.

Of these 201 operations, 74 (37\%) cases failed one or more of the examination criteria (Table 1). Nineteen patients $(9.5 \%)$ had deterioration in their corrected visual acuity on day 1 . Corneal oedema was the main reason for deterioration in vision post-operatively and the mean reduction was by 2 Snellen chart lines. However, by the 3 week clinic review all cases met the criteria for visual acuity with the exception of one case of corneal endothelial decompensation secondary to Fuchs' endothelial dystrophy. Visual outcome on the first postoperative day did not influence the routine management.

Twenty-six (13\%) patients were noted to have corneal oedema following surgery and only 8 of these had intraocular pressure above $30 \mathrm{mmHg}$. All these cases had recovered by the 3 week outpatient appointment. The management at day 1 did not change for corneal oedema unless associated with raised intraocular pressure. Eight (4\%) patients had more than $2+$ of cells in the anterior chamber and there were no hyphaemas. All of these had resolved by the 3 week outpatient appointment and did not result in a change in standard management. One patient had an eccentric pupil post-operatively with no obvious cause; he did not receive any additional treatment.

A total of 12 patients were noted to have raised intraocular pressure on the first day $(>30 \mathrm{mmHg}$ ). The range of pressures was from 30 to $50 \mathrm{mmHg}$. In all cases treatment was employed either by the use of oral slowrelease acetazolamide $250 \mathrm{mg}$, or reopening of the paracentesis with a glass rod. All intraocular pressures were less than $30 \mathrm{mmHg}$ at the 3 week review.

\section{Discussion}

Whitefield et al. ${ }^{4}$ performed a prospective study of 100 patients undergoing routine phacoemulsification and concluded that the first day follow-up could be abandoned. In our retrospective study involving twice as many patients we conclude differently, believing it to be unsafe to abandon the first day review unless ocular hypertension is controlled.

We have illustrated that the majority of patients do not have their management altered as a result of the first day post-operative visit. The visual acuity at day 1 was shown to be a poor prognostic indicator of the final vision obtained in the weeks following cataract surgery. Visual rehabilitation was largely attributable to spontaneous recovery of the corneal endothelium. We found that minimal activity in the anterior chamber following surgery was not uncommon following cataract extraction.

Further management was only implemented in the 12 patients $(6 \%)$ who had an intraocular pressure above 30 $\mathrm{mmHg}$. Intraocular pressure rise post-operatively was described by Rich et al., ${ }^{5}$ who found that it reached an average peak of $39.3 \mathrm{mmHg}$ at a mean time of $6.8 \mathrm{~h}$ following surgery. The pressure then rapidly decreased to an average of $23.1 \mathrm{mmHg}$ at $24 \mathrm{~h}$. The pressure rise is therefore seen to be self-limiting. However, some

Table 1. Number of patients fulfilling the five examination criteria $(\mathrm{n}=201)$

\begin{tabular}{llrr}
\hline Criteria & \multicolumn{1}{c}{ Day 1 } & Three weeks & Subsequent visits \\
\hline Improvement or no change in visual acuity & $90.5 \%(182)$ & $99.5 \%(200)$ & $99.5 \%(200)$ \\
Clear cornea & $87 \%(175)$ & $100 \%(201)$ & $100 \%(201)$ \\
Less than 2+ of cells in the anterior chamber & $96 \%(193)$ & $100 \%(201)$ & $100 \%(201)$ \\
A centred pupil & $99.5 \%(200)$ & $99.5 \%(200)$ & $99.5 \%(200)$ \\
Intraocular pressure of less than 30 mmHg & $94 \%(189)$ & $100 \% \quad(201)$ & $100 \% \quad(201)$ \\
\hline
\end{tabular}


patients will still have a high pressure after $24 \mathrm{~h}$. Further randomised prospective studies need to be performed to determine whether these pressures would resolve spontaneously with time or whether they have significance for long-term visual rehabilitation.

Patients with raised intraocular pressure frequently complain of headaches and nausea that do not resolve with oral analgesia, although these symptoms rapidly improve with oral acetazolamide. The first day review allows early identification and treatment of these cases. However, if the excessive pressure rise were avoided by the continued practice of careful removal of the viscoelastic at surgery and the use of an ocular hypotensive agent immediately following surgery it may now be justifiable to abandon the first day review. The patient could be assessed on the ward before returning home and given advice on removal of the eye shield and application of post-operative drops. The first and final outpatient appointment would be at 3 weeks, with an open door policy to review cataract patients in the interim. In this way the cataract operation could be managed in the three-episode model discussed by Ionides and Claoué. ${ }^{6}$ Thus day surgery would become a practical option for more patients.

\section{References}

1. Emery JM, Wilhelmus KA, Rosenberg S. Complications of phakoemulsification. Ophthalmology 1978;85:141-50.

2. Strong NP, Wigmore W, Smithson S, Rhodes S, Woodruff G, Rosenthal AR. Daycase cataract surgery. Br J Ophthalmol 1991;75:731-3.

3. Guidelines for cataract surgery. London: Royal College of Ophthalmologists, 1995.

4. Whitefield L, Crowston J, Little BC. First day follow up for routine phakoemulsification? Br J Ophthalmol 1996;80:148-50.

5. Rich WJ, Radtke ND, Cohen BE. Early ocular hypertension after cataract extraction. Br J Ophthalmol 1974;58:725.

6. Ionides A, Claoue C. Resource management of cataract patients: can visual rehabilitation be achieved in three visits? J Cataract Refract Surg 1996;22:717-20. 